\title{
An unusual case of nasal sarcoidosis
}

Pezier, T F ; Soyka, M B ; Holzmann, D

\begin{abstract}
Subcutaneous sarcoidosis is rare. As such, clinicians need a high index of suspicion when examining patients presenting with cosmetically unsightly lumps on the face. Sino-nasal manifestations of the disease should be sought with an endo-nasal examination, but definitive diagnosis requires histology. Even with good biopsy samples, the diagnosis may be elusive. We present a case from our clinic, which despite multiple biopsies, took almost 4 years to diagnose. Level of Evidence: Level V, diagnostic study.
\end{abstract}

DOI: https://doi.org/10.1007/s00238-013-0871-y

Posted at the Zurich Open Repository and Archive, University of Zurich ZORA URL: https://doi.org/10.5167/uzh-89890

Journal Article

Published Version

Originally published at:

Pezier, T F; Soyka, M B; Holzmann, D (2013). An unusual case of nasal sarcoidosis. European Journal of Plastic Surgery, 36:719-722.

DOI: https://doi.org/10.1007/s00238-013-0871-y 


\title{
An unusual case of nasal sarcoidosis
}

\author{
Thomas F. Pezier • Michael B. Soyka • David Holzmann
}

Received: 15 May 2013 / Accepted: 26 June 2013 / Published online: 16 July 2013

(C) Springer-Verlag Berlin Heidelberg 2013

\begin{abstract}
Subcutaneous sarcoidosis is rare. As such, clinicians need a high index of suspicion when examining patients presenting with cosmetically unsightly lumps on the face. Sino-nasal manifestations of the disease should be sought with an endo-nasal examination, but definitive diagnosis requires histology. Even with good biopsy samples, the diagnosis may be elusive. We present a case from our clinic, which despite multiple biopsies, took almost 4 years to diagnose.

Level of Evidence: Level V, diagnostic study.
\end{abstract}

Keywords Sarcoidosis - Granuloma - Rhinoplasty · TAP deficiency $\cdot$ Eosinophilic angiocentric fibrosis

\section{Introduction}

There is a growing trend in recent years for patients to present to their surgeon with primarily cosmetic concerns. These may be somewhat hidden behind feigned functional concerns (e.g. my glasses do not fit my nose or wearing my glasses is painful), but careful questioning often elucidates the patient's key concern. Patients may be extremely surprised to learn that their cosmetic concern is actually the local manifestation of a systemic disease. Furthermore, some of these systemic diseases carry a substantial social taboo

T. F. Pezier $(\bowtie) \cdot$ M. B. Soyka $\cdot$ D. Holzmann

Department of Otorhinolarnygology, Head and Neck Surgery, University Hospital Zurich, Frauenklinikstrasse 24, 8091 Zurich, Switzerland

e-mail: tfrpezier@gmail.com (e.g. the syphilitic saddle nose) or a life-changing prognosis (e.g. malignant melanoma).

We present a case of a fluctuating nasal lump which defied diagnosis and treatment for several years and which, besides the medical management, required sensitive psychosocial skills.

\section{Case report}

A 38-year-old lady was referred to our ENT clinic in March 2008 with a persistent swelling on the right side of her nose preventing her from wearing her glasses comfortably (Fig. 1). The patient had no other symptoms, and full ENT examination including nasal endoscopy together with routine blood tests was normal. The lump itself was hard, pain free and slowly growing. There were no overlying skin changes. A CT scan (Fig. 2) showed focal demineralization of the right nasal bone and surrounding soft tissue swelling, consistent with chronic inflammation.

In order to rule out malignancy and for further diagnostic work-up, the patient underwent a punch biopsy (including bone) under local anaesthetic. Histological examination showed granulomatous chronic inflammation with focal necrosis but without signs of vasculitis. The pathologist, however, was unable to give a specific diagnosis. Despite negative PCR for mycobacterium (including tuberculosis and leprosy) and negative staining for fungi, an infectious agent was felt to be the cause as sarcoid was felt unlikely to cause focal necrosis. Immunological screening effectively ruled out TAP deficiency (a peptide transport complex associated with antigen presentation) and vasculitides. 
Fig. 1 Clinical photo showing the right-sided nasal lesion

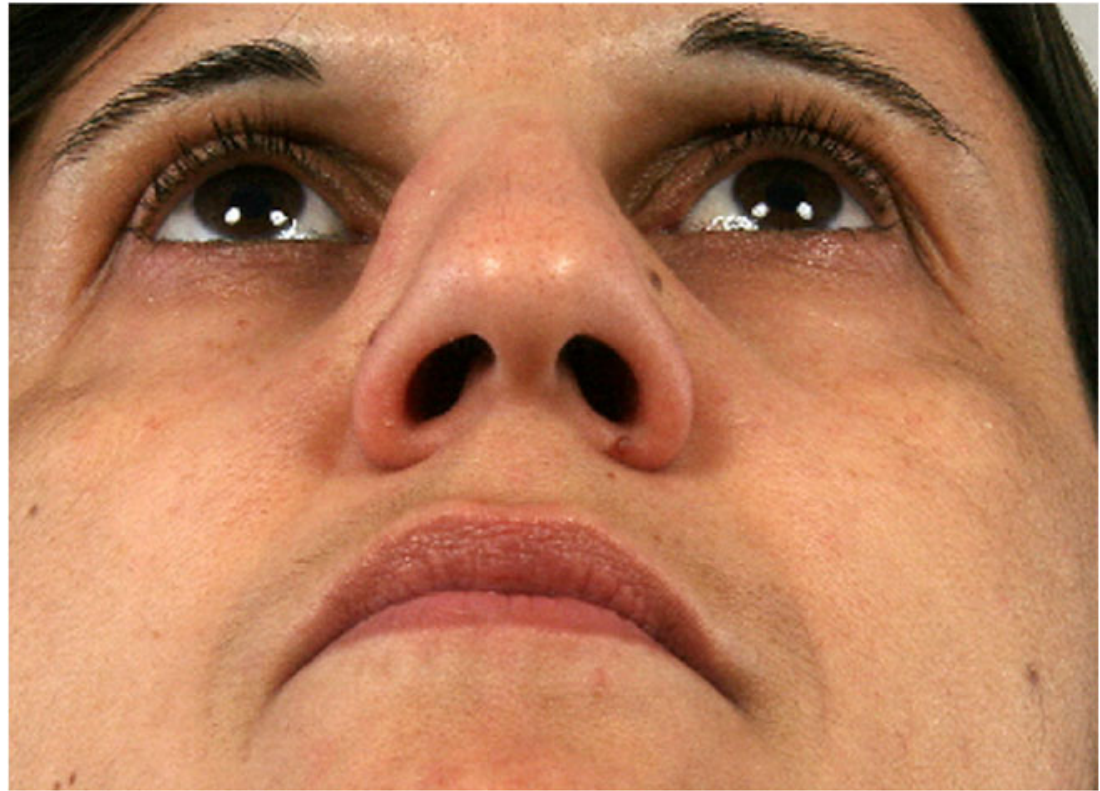

Following the biopsy, the lump became smaller, and the patient's general health remained excellent. A further biopsy was recommended for further testing (microbiological, mycobacteriological, parasitology and mycology), but the patient declined. She attended regular follow-up, and when a year later the mass began to grow again, she agreed to biopsy. A transcartilaginous biopsy was performed under general anaesthetic. This again showed similar, non-specific findings despite ex- tensive testing (PAS, Ziehl-Nielson, Grocott, BrownBrenn). A chest $\mathrm{x}$-ray showed no typical sarcoid lesions, and lung spirometry was normal.

Finally, in January 2012, the swelling became bilateral, and the decision was made for explorative rhinoplasty. A closed technique was used, and bluish, spongy bone was excised. Histopathologically, this again showed necrotic granulomatous change, and the possibility of eosinophilic angiocentric fibrosis was raised. A
Fig. 2 CT showing bony demineralization

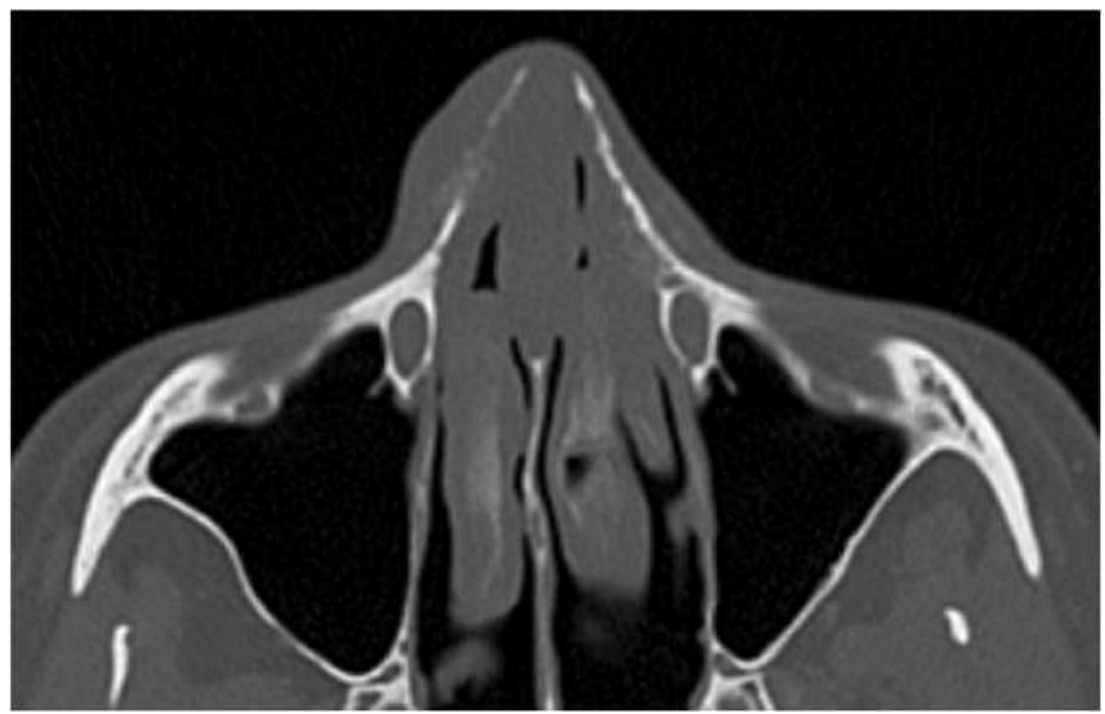


chest CT showed bilateral lymphadenopathy consistent with sarcoidosis. Bronchoscopy with ultrasound confirmed these findings and showed no lung parenchymal changes.

After interdisciplinary discussion with the pathologists, pulmonologists, rheumatologists and ENT clinicians, a diagnosis of sarcoid stage 1 was made. Systemic steroid therapy was recommended, but the patient declined. She is still in regular follow-up and has no further complaints since the surgery.

\section{Discussion}

Sarcoidosis is a poorly understood multisystem disease. The prevalence varies from $<1$ to 40 per 100,000 depending on location [1]. It is characterized by noncaseating granulomas which can affect many different organ systems [2]. Ninety percent of cases have lung involvement, and typically, patients are diagnosed by chest $\mathrm{x}$-ray with pulmonologists being the treating physicians. Roughly $1 \%$ of patients present with sino-nasal symptoms.

Subcutaneous sarcoidosis is rare and was first reported in 1904 [3]. Findings may mimic other diseases, and this, coupled to the fact that up to $80 \%$ of patients with subcutaneous sarcoid have no signs of systemic sarcoidosis [4], makes clinical diagnosis challenging. The few case reports in the literature show a preponderance for middle-aged women [5].

On suspicion of sarcoid, patients should undergo a thorough full body clinical examination. Manifestations of sarcoid in the nose [5] include red intra-nasal plaques, obstruction, crusting and epistaxis as well as cartilage destruction with resultant widening of the nasal bridge and saddle nose deformity. Consideration should be given for early pulmonological/rheumatological consult.

Simple, minimally invasive tests such as blood tests (serum ACE, hypercalcaemia, hypercalciuria) [6], fundoscopy and plain chest $\mathrm{x}$-ray can be performed. Though these may be suggestive, none of these tests has particularly good sensitivity or specificity. More invasive tests such as fine-needle aspiration cytology can often be inconclusive. In cosmetically sensitive areas, tissue biopsy can result in scars and contour changes, but this may be necessary for histopathological diagnosis [7].

Under the microscope, sarcoid masses are composed of epithelioid granulomas, giant cells and hyalinosis. Focal necrosis is not typical, and testing for other granulomatous diseases, notably tuberculosis and leprosy, should be performed. The possibility of vasculitides and chronic fungal infection should also be tested. If results are equivocal, exotic conditions such as TAP deficiency [8] and eosinophilic angiocentric fibrosis [9] should be considered.

Currently, there is no cure for sarcoidosis. Immune suppression is frequently used, especially in patients with extensive lung parenchymal changes. In well-localized lesions, locally injected preparations [10] can sometimes be used. Surgical excision can remove a sarcoid mass but is not considered curative.

\section{Conclusions}

Sarcoid is typically a multisystem disease but may present with discrete lesions. A high index of suspicion together with early consult from pulmonologists and rheumatologists can help make the diagnosis, which otherwise, despite biopsy, can remain elusive. Facial surgeons should be aware that cosmetically unsightly lumps on the face may be manifestations of complex systemic diseases.

\section{Conflict of interest None}

Patient consent The patient provided written consent for the use of her images.

\section{References}

1. Vainsencher D, Winkelmann RK (1984) Subcutaneous sarcoidosis. Arch Dermatol 120(8):1028-1031

2. Bauer MP, Brouwer PA, Smit VT, Tamsma JT (2007) The challenges of extrapulmonary presentations of sarcoidosis: a case report and review of diagnostic strategies. Eur J Intern Med 18(2):152-154

3. Darier JRG (1904) Des sarcoïdes sou-cutanées. Contribution à l'étude des tuberculides ou tuberculoses attenuées de l'hypoderme. Ann Dermatol Syphilol 5:144-149

4. Kerner M, Ziv M, Abu-Raya F, Horowitz E, Rozenman D (2008) Subcutaneous sarcoidosis with neurological involvement: an unusual combination. Isr Med Assoc J 10(6):428430

5. Aloulah M, Manes RP, Ng YH, Fitzgerald JE, Glazer CS, Ryan MW, Marple BF, Batra PS (2013) Sinonasal manifestations of sarcoidosis: a single institution experience with 38 cases. Int Forum Allergy Rhinol. doi:10.1002/alr.21142

6. Costabel U, Teschler H (1997) Biochemical changes in sarcoidosis. Clin Chest Med 18(4):827-842

7. Hunninghake GW, Costabel U, Ando M et al (1999) ATS/ERS/ WASOG statement on sarcoidosis. American Thoracic Society/ European Respiratory Society/World Association of Sarcoidosis 
and Other Granulomatous Disorders. Sarcoidosis Vasc Diffuse Lung Dis 16(2):149-173

8. Gadola SD, Moins-Teisserenc HT, Trowsdale J, Gross WL, Cerundolo V (2000) TAP deficiency syndrome. Clin Exp Immunol 121(2):173-178
9. Magro CM, Dyrsen M (2008) Angiocentric lesions of the head and neck. Head Neck Pathol 2(2):116-130

10. van Griethuysen J, Kuchai R, Taghi AS, Saleh HA (2012) Nasal sarcoidosis: a cause for a medical rhinoplasty? J Laryngol Otol 126(10):1073-1076 